\title{
Intracoronary Imaging and Histopathology of Late Phase In-Stent Restenosis after Coronary Stent Implantation
}

\section{Shigenori Ito,, ${ }^{1,2}$ Kosuke Nakasuka, ${ }^{1}$ Satoru Sekimoto, ${ }^{1}$ Kazuyuki Miyata, ${ }^{1}$ Masahiko Inomata, ${ }^{1}$ Takayuki Yoshida, ${ }^{1}$ Nozomu Tamai, ${ }^{1}$ Tomoaki Saeki, ${ }^{1}$ Shin Suzuki, Yoshimasa Murakami, ${ }^{1}$ and Koichi Sato ${ }^{1}$}

${ }^{1}$ Division of Cardiology, Nagoya City East Medical Center, Nagoya, Aichi 464-8547, Japan

21-2-23 Wakamizu, Chikusa, Nagoya, Aichi 464-8547, Japan

Correspondence should be addressed to Shigenori Ito, sito@higashi-hosp.jp

Received 4 October 2012; Accepted 20 October 2012

Academic Editors: D. Guidolin and C.-C. Wu

Copyright () 2012 Shigenori Ito et al. This is an open access article distributed under the Creative Commons Attribution License, which permits unrestricted use, distribution, and reproduction in any medium, provided the original work is properly cited.

In the field of coronary artery stenting, restenosis issue has been almost resolved after development of drug-eluting stents (DESs). In-stent restenosis (ISR) is generally considered to be a stable process, with an early peak in intimal hyperplasia followed by a quiescent period beginning 1 year after stent implantation. However, recently, extended-term problem (over 1 year) in both baremetal stents (BMSs) and DES has been reported. This phenomenon seems to be different from early ISR within one year in terms of its mechanism, prevention, and treatment. In this paper, we discuss this topic from the standpoint of intracoronary imagings and histopathology, referencing several interesting cases we experienced.

\section{Introduction}

In the field of coronary artery stenting, restenosis issue has been almost resolved after development of drug-eluting stents (DESs). In-stent restenosis (ISR) is generally considered to be a stable process, with an early peak in intimal hyperplasia followed by a quiescent period beginning 1 year after stent implantation. However, recently, extended term problem (over 1 year) in both bare-metal stents (BMSs) and DES has been reported. Very late stent thrombosis (over 1 year) is a challenging problem in patients who underwent DES implantation. It may occur at low incidence with $0.2 \%$ $0.6 \%$ yearly; however, it could be lethal if it occurs. On the other hand, late ISR also is another problem in both DES and BMS implantation. This phenomenon seems to be different from early ISR within one year in terms of its mechanism, prevention, and treatment. In this paper, we discuss this topic from the standpoint of intracoronary imagings and histopathology, referencing several interesting cases we experienced.

\section{Clinical Course of ISR after Bare-Metal Stent (BMS) Implantation}

In spite of the efficacy and stability of coronary bare-metal stents in the early-and medium-term phase, the long-term safety of implanted stent remains controversial. Choussat et al. [1] reported that clinical stability of the stented target site is at $8-10$ years after coronary stenting. By contrast, Kimura et al. [2] reported that 7-11 years of angiographic followup demonstrated late luminal renarrowing beyond 4 years, which did not necessarily require target lesion PCI. They also reported that the long-term luminal response after coronary stenting was triphasic: an early restenotic phase (until 6 months), an intermediate-term regression phase (from 6 months to 3 years), and a late renarrowing phase (beyond 4 years). A late renarrowing represents the late ISR.

\section{Late In-Stent Restenosis after BMS}

The previous study [3] and our first case (Figures 1, 2, 3, 4, 5, 6 , and 7) [4] clearly demonstrated that new atherosclerotic 


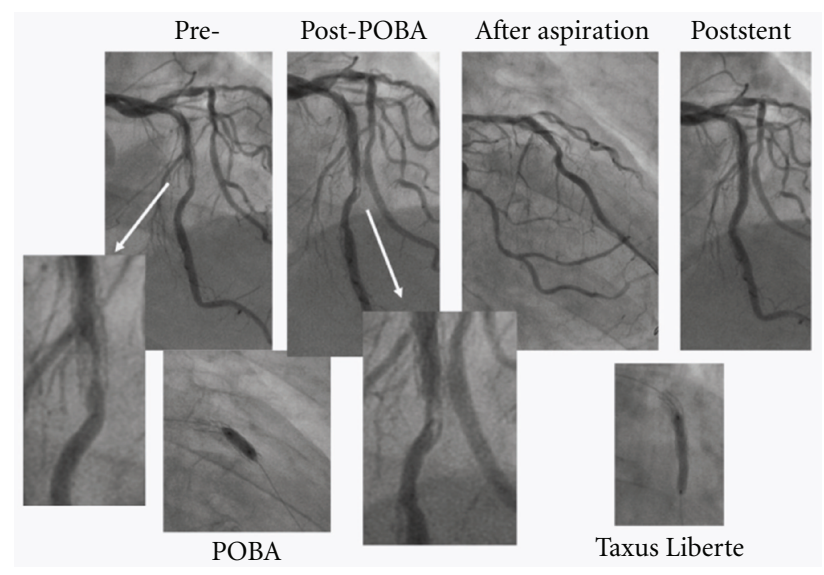

FIGURE 1: Coronary angiograms during index percutaneous coronary intervention. Balloon angioplasty was performed for in-stent restenosis in the mid-left anterior descending coronary artery. After balloon dilatation, a filling defect was seen just distal to the stent with resultant improvement of in-stent stenosis. After aspiration, the defect was not angiographically visible. A $2.5 \times 20 \mathrm{~mm}$ Taxus Liberte stent was deployed to cover the in-stent restenotic lesion, and the result was good. (From J Invasive Cardiol 23: E86-E91, 2011 with permission).
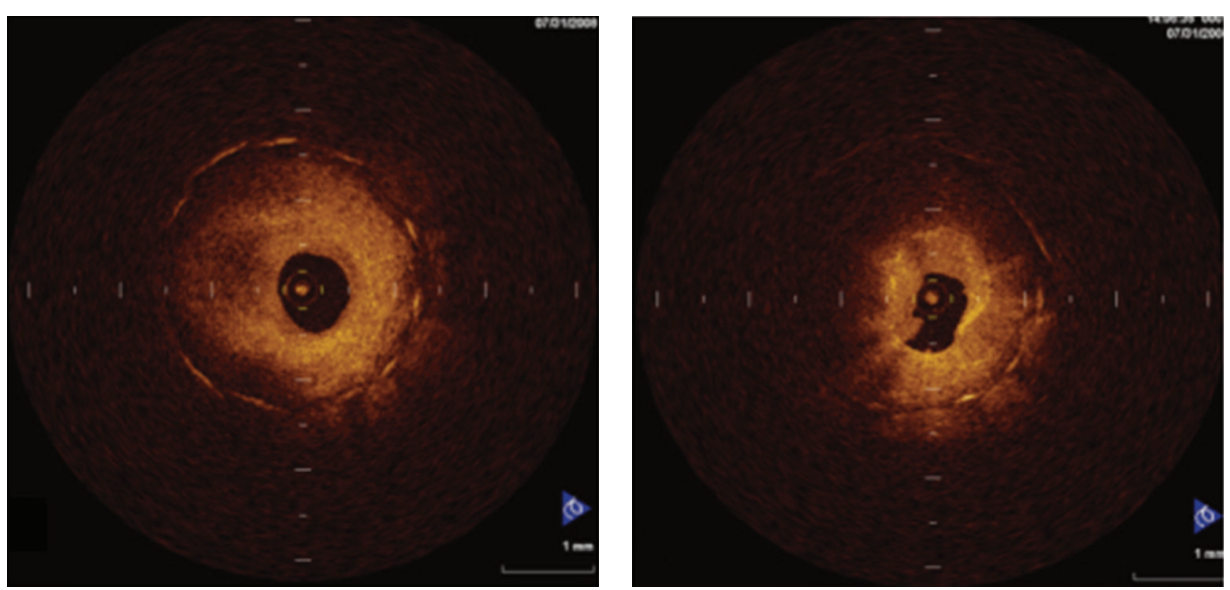

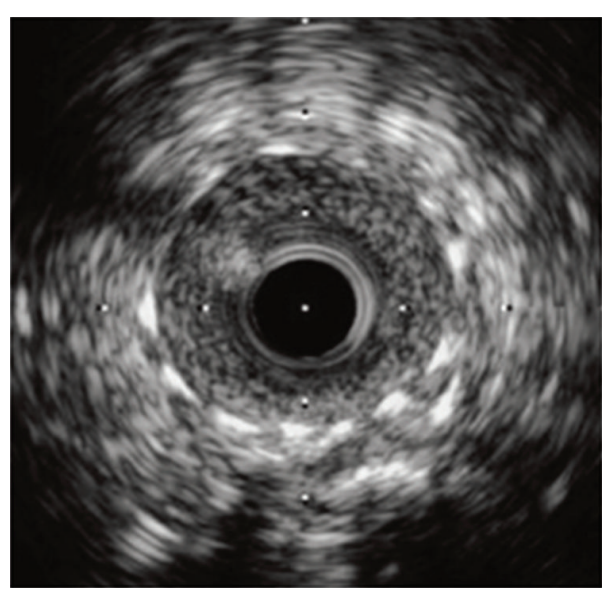

(a)

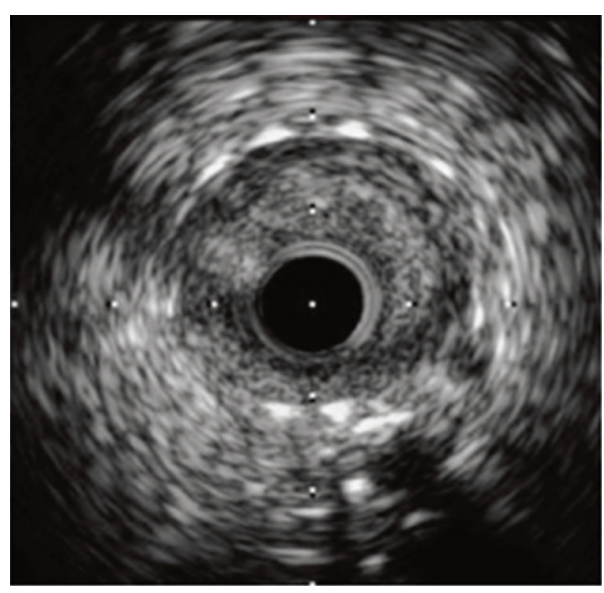

(b)

FIGURE 2: Optical coherence tomography (OCT) and intravascular ultrasound (IVUS) findings of in-stent restenosis before balloon angioplasty. (a) OCT showed homogeneous high intensity in the distal segment. IVUS revealed a homogeneous and slightly hyperechoic material. (b) High intensity accompanied by attenuation was observed in the proximal segment by OCT. Peristent area was hypoechoic by IVUS; however, there was no clear difference between (a) and (b) compared to OCT. (From J Invasive Cardiol 23: E86-E91, 2011 with permission). 

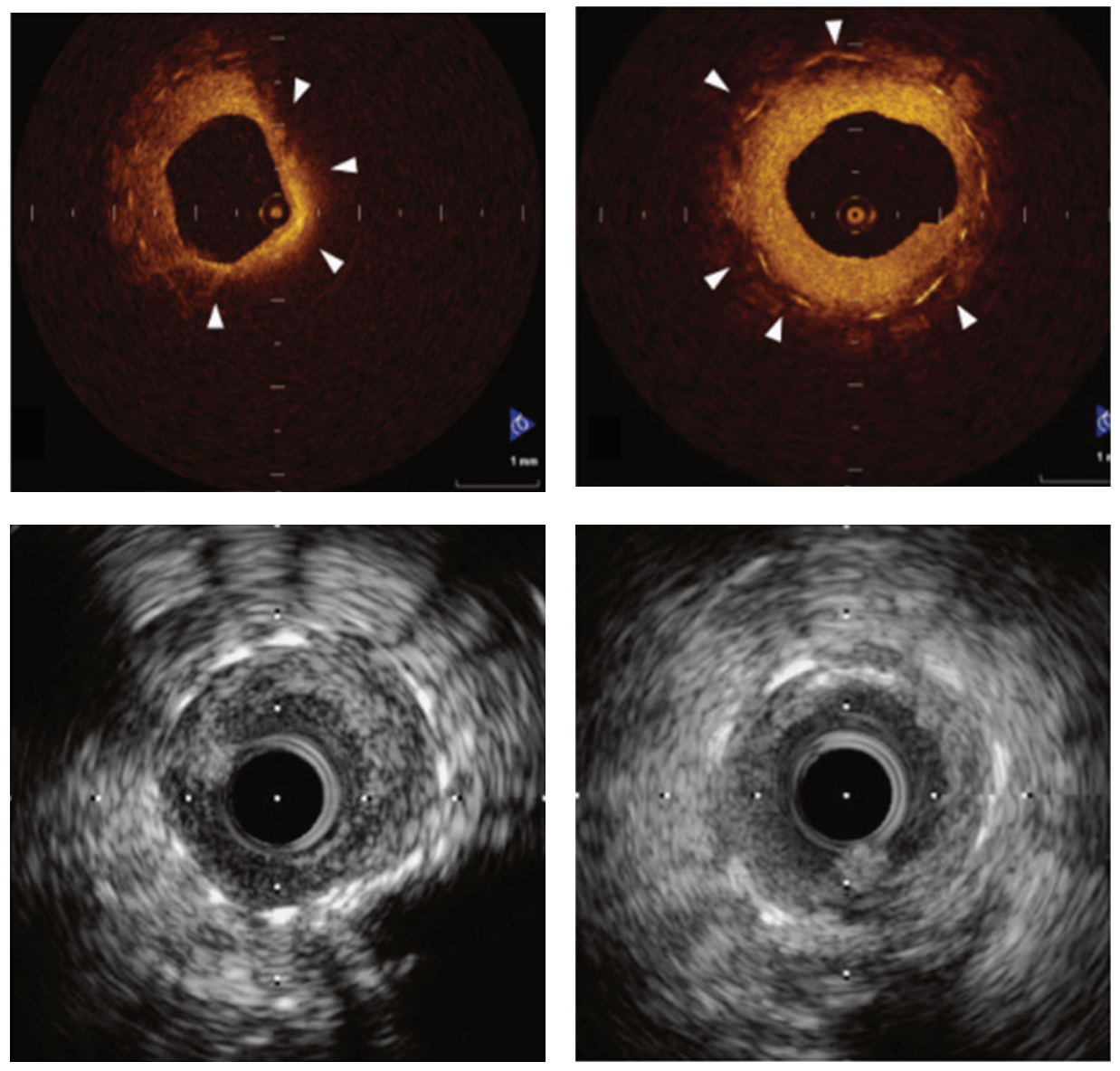

(a)

(b)

Figure 3: (a) Thin-cap fibroatheroma without significant stenosis proximal to the in-stent restenotic lesion. (b) Low signals surrounding some stent struts. Intravascular ultrasound could not identify the thin-cap fibroatheroma or low signals surrounding the stent struts. (From J Invasive Cardiol 23: E86-E91, 2011 with permission).

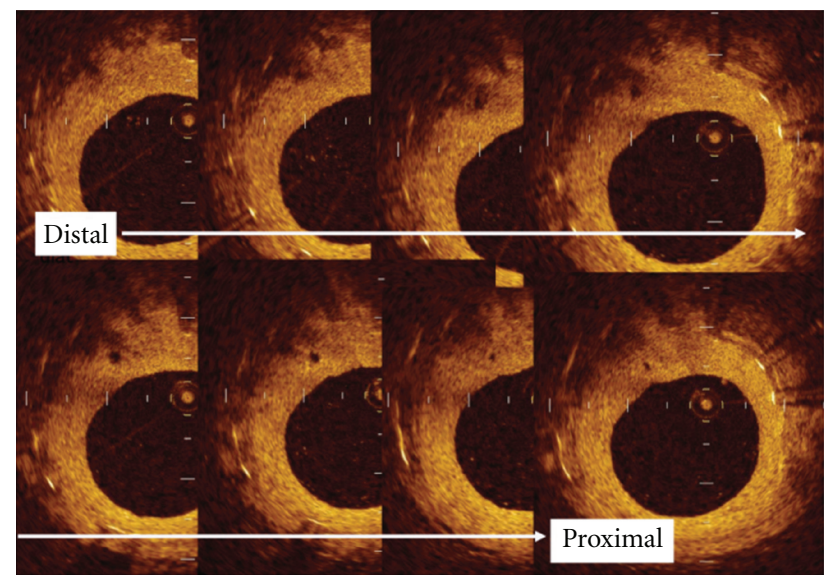

FIGURE 4: Frame-by-frame OCT images showing a microvessel. There was a tubular structure (diameter $=100 \mu \mathrm{m})$ expanding the peristent to the intima suggesting neovascularization observed $3 \mathrm{~mm}$ distal to the proximal edge. (From J Invasive Cardiol 23: E86-E91, 2011 with permission). 

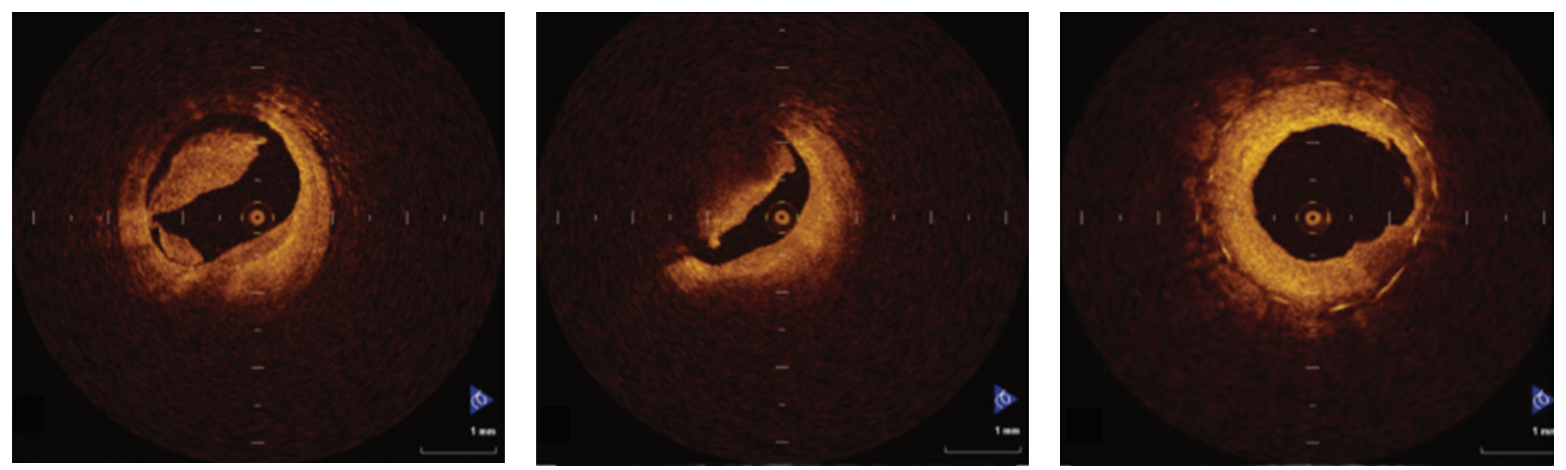

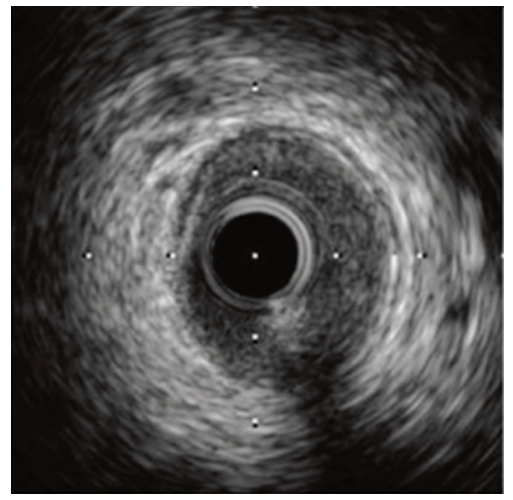

(a)

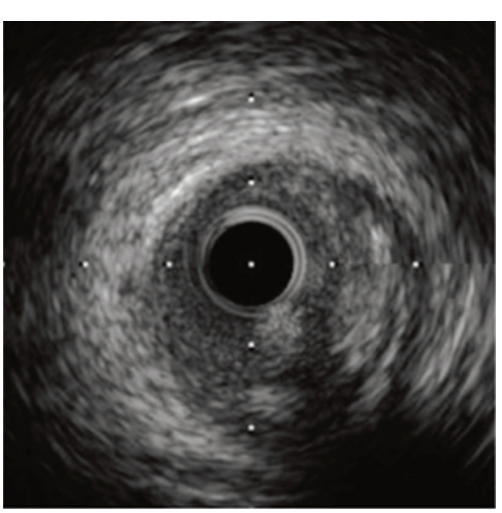

(b)

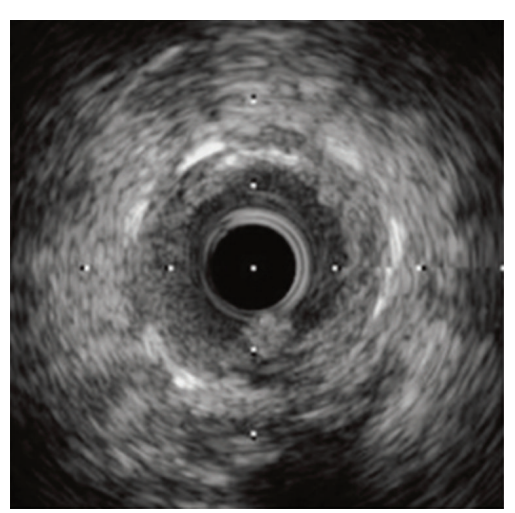

(c)

FIGURE 5: Optical coherence tomographic findings of in-stent restenosis after balloon angioplasty. (a) Distal part of in-stent restenosis. The distal half of the material had a high intensity homogenous signal. (b) Proximal part of in-stent restenosis. The proximal half of the retrieved material showed superficial high-intensity with attenuation, suggesting that they were composed of different components. (c) Resultant tissues showed heterogeneous plaque containing partial low signals. (From J Invasive Cardiol 23: E86-E91, 2011 with permission).

progression is observed in late ISR, especially in extended late phase ISR (over 5 years) after BMS implantation. In our case [4], pathologically the retrieved tissue appeared to be double-layered facing the healed neointimal layer inside the implanted stents, suggesting that accumulation of the tissue might have occurred in 2 stages. In fact, the ISR occurred with a time lag of over ten years after the last angiography at another hospital that showed moderate intimal hyperplasia with 50\% angiographic stenosis. The detection of macrophages by CD 68 staining and pleomorphic smooth muscle cells (SMCs) by anti-SMC alpha-actin staining might suggest that activation of the intimal hyperplasia may have restarted or continued once the intimal hyperplasia had remained stabilized for over 10 years following BMS implantation. Pathologic studies have demonstrated that mechanism of very late ISR is quite different from that of early ISR. Probably, neoatherosclerosis could be a main cause of ISR in patients with late ISR after BMS implantation.

\section{OCT and Plaque Characteristics in ISR}

OCT has been the most powerful tool to delineate the restenotic tissue imaging and to classify the tissue components. On the other hand, IVUS has some limitations in visualizing its complex tissue structure as can be documented by histopathology. In 2009, Gonzalo et al. [5] evaluated the morphologic characteristics of ISR by OCT. They classified the ISR tissue in terms of restenotic tissue structure (homogeneous, heterogeneous, and layered), restenotic tissue backscatter (high, low), presence or absence of microvessels, lumen shape (regular, irregular), and presence of intraluminal material in 24 patients with 25 ISR tissue (DES 84\% BMS 16\%). Tissue structure was layered in $52 \%$, homogeneous in $28 \%$, and heterogeneous in $20 \%$. The predominant backscatter was high in $72 \%$. Stent implanted $\leq 12$ months ago had more frequently restenotic tissue with layered appearance ( $84.6 \%$ versus $16.7 \%, P=0.003)$. Their classification has been adapted in the following reports about the OCT findings of ISR.

\section{Intracoronary Imagings of ISR after BMS Implantation}

As a relatively rare cause of early ISR, although probably common in very late ISR, after BMS implantation, neoatherosclerosis has been reported in the extended longterm followup periods in two pathological $[6,7]$ and an OCT study [8]. We also successfully obtained both IVUS and OCT images of such a ruptured plaque in an acute coronary syndrome case [9] with late ISR 8 years after BMS implantation. Reflecting the difference in their mechanism, the image characteristics of ISR after BMS may be different 


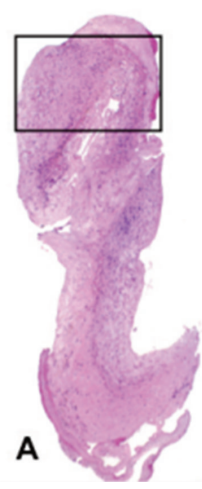

(a)

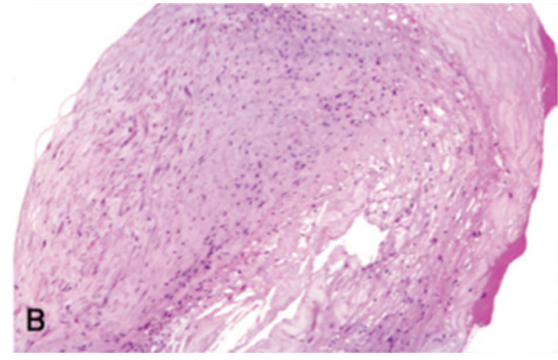

(b)

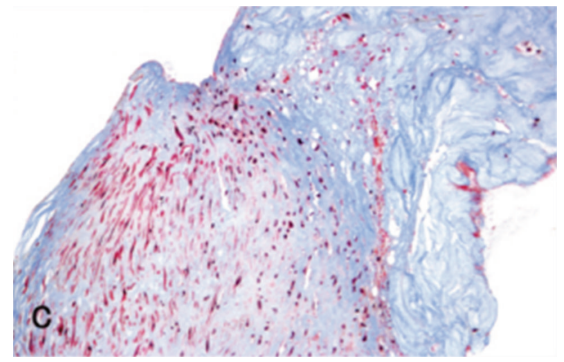

(c)

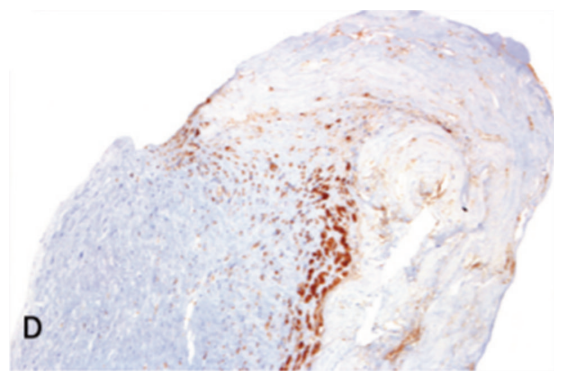

(d)

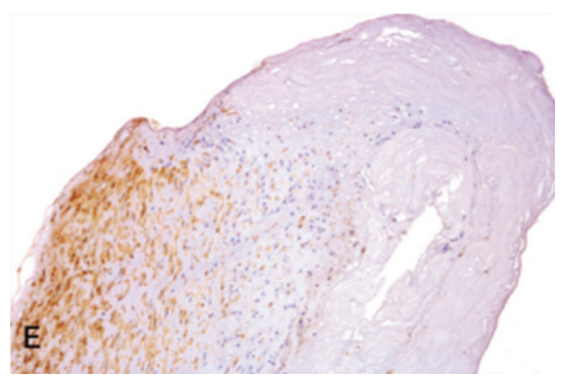

(e)

Figure 6: (a) Hematoxylin-eosin stain. The entire body of this material seemed to be composed of two layers. box in (a) is magnified in Figures (b)-(e). (b) Hematoxylin-eosin stain. (c) Masons trichrome stain. (d) immunoreactivity to CD 68. (e)Immunoreactivity to SMC $\alpha$-actin. (From J Invasive Cardiol 23: E86-E91, 2011 with permission).

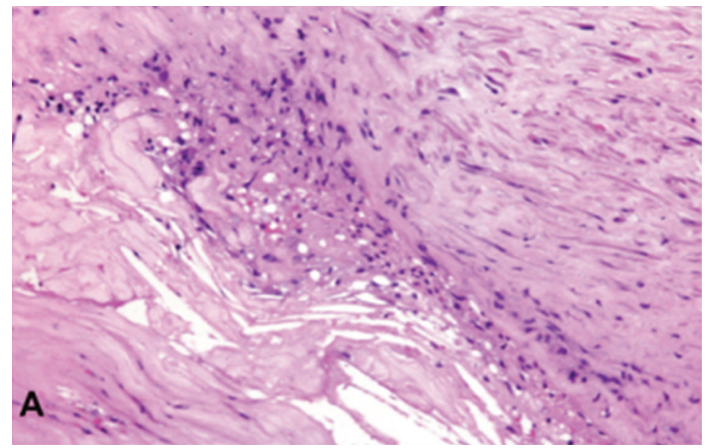

(a)

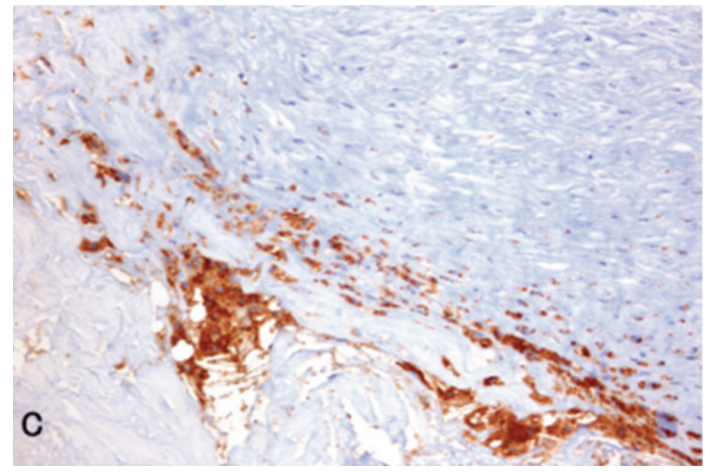

(c)

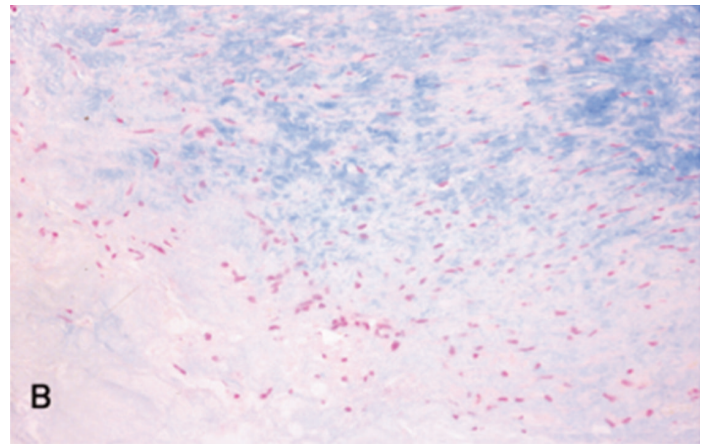

(b)

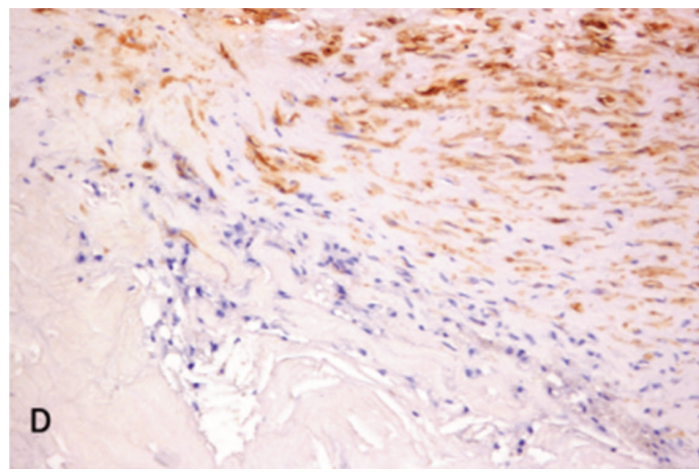

(d)

Figure 7: Microscopic findings (higher magnification). (a) Hematoxylin-eosin stain. (b) Alcian blue stain. (c) Immunoreactivity to CD 68. (d) Immunoreactivity to SMC $\alpha$-actin. Fibrous collagenous plaque is adjacent to the lesion where polymorphic SMCs proliferate with rich proteoglycan at the background. (From J Invasive Cardiol 23: E86-E91, 2011 with permission). 


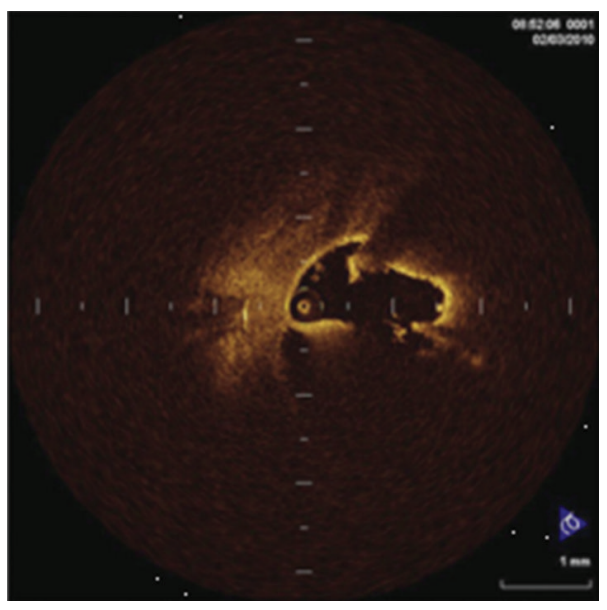

(a)

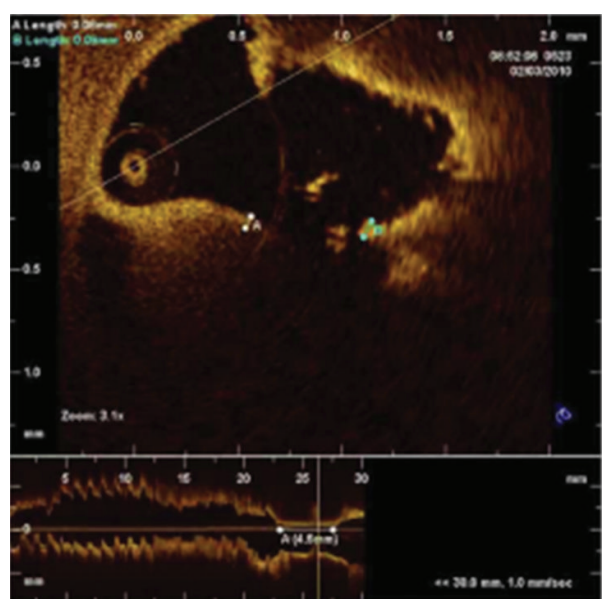

(b)

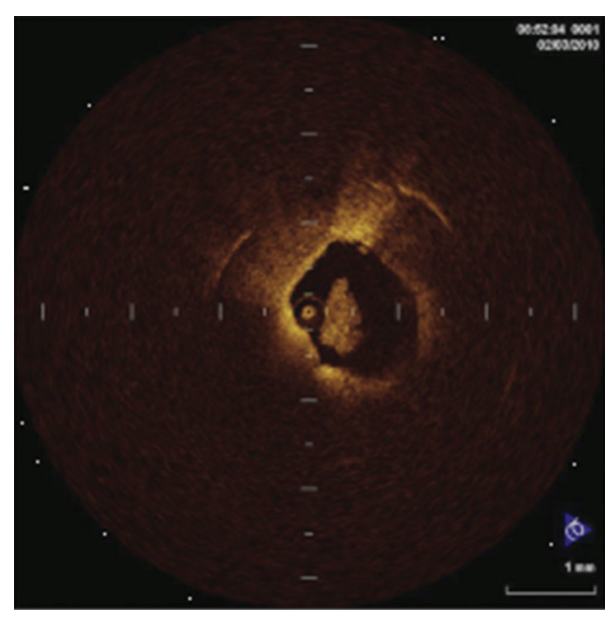

(c)

FIGURE 8: Optical coherence tomography findings. (a) Ruptured thin-cap fibroatheroma with homogeneous low intensity signals. (b) Measurements of thin fibrous cap ((a): $60 \mu \mathrm{m},(\mathrm{b}): 90 \mu \mathrm{m})$. (c) White thrombus can be detected on the thin fibrous cap near the ruptured site. (From JC Cases 5: e1-e3, 2012 with permission).

between the early phase and extended late phase (usually over 5 years) [10]. Early stage ISR is characterized by both IVUS and OCT as homogenous high intensity signals. On the other hand, extended late phase ISR shows various signal patterns in plaque composition [10]. The proportion of cross-sections with heterogeneous intima in the entire stent was significantly higher in the very late ISR (over 5 years) group compared to the early ISR (within the first year) group $(60.5 \pm 28.5 \%$ versus $5.8 \pm 11.5 \%, P<0.0001)$, with heterogeneous intima being more frequently observed at the minimum lumen area site in the very late-ISR group (90.7\% versus $17.9 \%$ ). This might imply that several types of tissue are formed during an extended followup period. The OCT findings in the very late ISR are similar to the morphology observed with typical native atherosclerotic lesions. That is, the signal-poor area with diffuse lumen border was similar to the OCT image of lipid-laden plaque in the atherosclerotic lesion. Our second case [9] (Figures 8 and 9) clearly shows the disruption of fibrous cap associated with white thrombus by OCT. Deep attenuation under a thin fibrous cap might be expressed as echolucent plaque by
IVUS, because the location of the plaque is almost identical between IVUS and OCT images in the same cross-section level. OCT was very useful, like it is for de novo vulnerable plaques, to evaluate vulnerable plaque that had formed in a previously implanted stent. On the other hand, tissue outside the stent struts could be visualized by IVUS. Combined use of OCT and IVUS was useful to understand the characteristics of a ruptured vulnerable plaque that had formed in a previously implanted bare-metal stent even after stabilization of neointimal hyperplasia. If such plaque rupture repeats silently in patients with coronary stents, tissue in the stent accumulates gradually leading to the severe stenosis like in this case.

\section{Late In-Stent Restenosis after DES Implantation}

The phenomenon of late ISR is rather infrequent, occurring in $2.6 \%$ of treated lesions [11], but has a steady annual rate after sirolimus-eluting stent (SES) implantation. The mechanisms of the late catch-up phenomenon are 
incompletely understood after DES implantation. Long-term inflammation has been reported after SES implantation by pathological observation in humans [12]. Since the sirolimus is eluted from the stent completely within about 120 days, this inflammation might be due to polymer toxicity or hypersensitivity. Recently, a couple of studies reported that the other cause of late ISR is neoatherosclerosis that formed in the stent $[6,8,13]$. As the other cause of SES late ISR, we encountered an interesting case [14] in which fibrin deposition was observed in the SES implanted 39 months ago. There is also a possibility that retrieved fibrin in this case had been formed during a previous rupture of neoatherosclerosis that formed in the stent $[6,8]$. In our third case (Figures 10 and 11), we speculated that inflammation and delayed healing with coronary endothelial dysfunction $[15,16]$ might be related to the fibrin thrombus formation. Also, thin-cap fibroatheroma (TCFA) was recognized in the short segment of the restenotic tissue suggesting the existence of neoatherosclerosis. On the other hand, Sawada et al. [17] recently reported that some subclinical intrastent thrombi might eventually result in DES ISR, and some restenosed DES neointima might be configured by thrombi. This possibility leads to the hypothesis that the CYP2C19*2 polymorphism is associated with DES restenosis as well as intrastent thrombus through a poor response to clopidogrel. Although the patient had taken both aspirin and ticlopidine, drug resistance should also be considered. Fujii et al. [18] also demonstrated the contribution of organized thrombus to in-stent restenosis after SES implantation by OCT findings 12 months after implantation. At the ISR site, OCT revealed a low-backscattering irregular mass protruding into the lumen. This finding also may suggest that the organized thrombus was the main component of restenotic tissue after SES implantation.

\section{Intracoronary Imagings of Late Phase ISR after DES Implantation}

Kang et al. [13] reported findings from OCT of in-stent neoatherosclerosis as a cause of DES failure in 50 patients (30 stable, 20 unstable angina) with 50 DES ISR lesions and intimal hyperplasia $>50 \%$ of stent area. Compared with DES $<20$ months after implantation, DES $\geq 20$ months after implantation had a higher incidence of TCFA-containing neointima (69\% versus $33 \%, P=0.012)$ and red thrombi (27\% versus o\%, $P=0.007)$. Furthermore, their study showed that patients with late DES-ISR were more likely to have an unstable clinical manifestation compared to those with early DES-ISR. We encountered the third case with typical presentation 6 years after SES implantation. As a second cause of ISR after SES, fibrin thrombus and fibrinoid are important. To date, there are no OCT criteria for organized thrombus and fibrinoid corresponding with histological validation [19]. However, in our very late ISR after SES [14], we speculated that low backscatter backward with a superficial high-signal fibrous cap, where stent struts were identified by OCT and low-echoic plaque by IVUS, might suggest fibrin thrombus. Special staining and immunohistological study showed that there was no massive

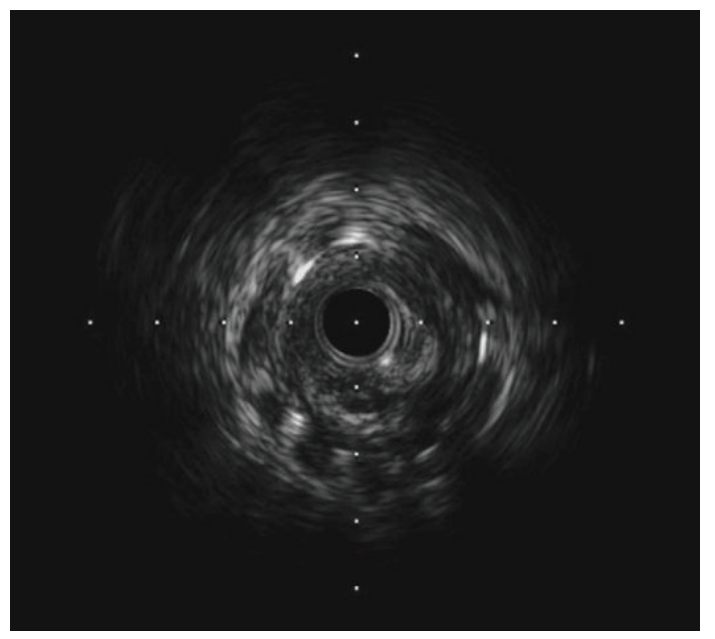

Figure 9: Intravascular ultrasound finding. Heterogeneous and mainly echolucent plaque can be seen nearly at the identical site of ruptured site detected by optical coherence tomography. There was also homogeneous echolucent appearance observed outside the stent struts. Obvious stent malapposition was not detected. (From JC Cases 5: e1-e3, 2012 with permission).

deposition of proteoglycans or smooth muscle cells. Because all restenotic tissue could not be retrieved by Filtrap in this case, it is impossible to determine the OCT and IVUS features of a fibrin thrombus definitely. It is sometimes difficult to discern fibrin from neoatherosclerosis. Massive fibrin accumulation is seen on OCT as a dark area without a clear border, which resembles necrotic core [20].

\section{Difference in Late Phase ISR between BMS and DES}

Nakazawa et al. investigated the pathology of neoatherosclerosis in human coronary stent implantation in 299 autopsy cases with a total of 406 lesions (197 BMS, 209 DES 103 SESs, and 106 paclitaxel-eluting stents) [6]. The incidence of neoatherosclerosis was significantly higher in DES lesions $(31 \%)$ than in BMS lesions $(16 \% ; P<0.001)$. The median stent duration with neoatherosclerosis was shorter in DES than BMS (DES, 420 days (interquartile range: 361 to 683 days); BMS, 2,160 days (interquartile range; 1,800 to 2,880 days), $P<0.001)$. Unstable lesions characterized as TCFA or plaque rupture were more frequent in BMS $(7.4 \%)$ than in DES (3.1\%, $P=0.17$ ), with relatively shorter implant duration for DES ( $1.5 \pm 0.4$ years) compared to BMS $(6.1 \pm 1.5$ years). Thus, they concluded that neoatherosclerosis is a frequent finding in DES and occurs earlier than in BMS [6]. The development of neoatherosclerosis may be yet another rare contributing factor to late stent thrombosis. Furthermore, this phenomenon manifest as stable in-stent restenosis before plaque rupture followed by stent thrombosis.

\section{Mechanism of Neoatherosclerosis in the Stents}

The pathological mechanism of new atherosclerotic plaque formation at extended long-period followup is not well 


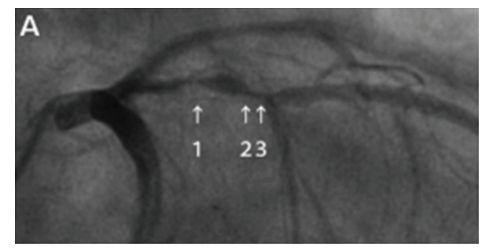

(a)

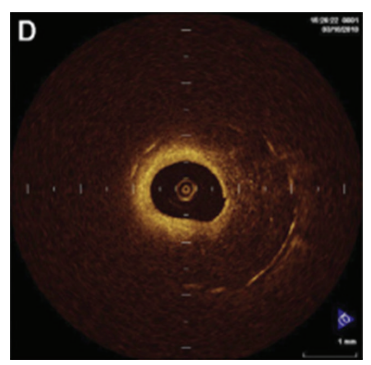

(d)

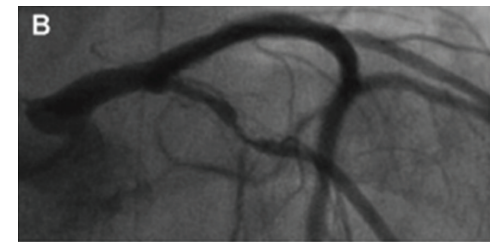

(b)

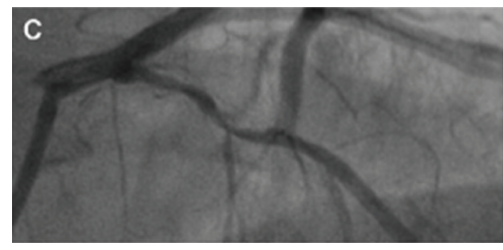

(c)

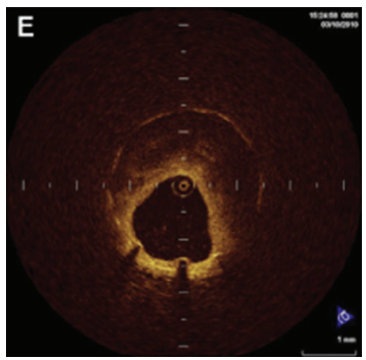

(e)

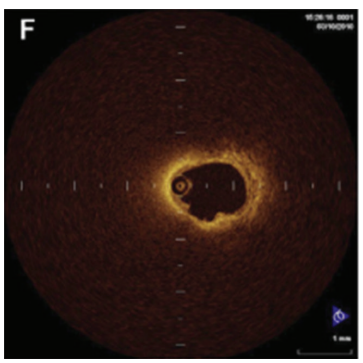

(f)

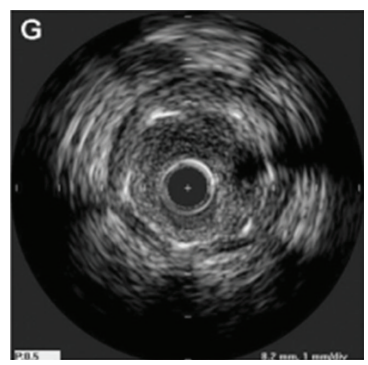

(g)

FIGURE 10: (a) Followup coronary angiography at 39 months (RAO caudal view). Arrows 1, 2, and 3 indicate corresponding cross-sections of the OCT findings in Figures (d)- (f). (b) Followup coronary angiography at 39 months (AP cranial view). (c) Control coronary angiography before PCI two months after the diagnostic coronary angiography (AP cranial view). There was no progression of stenosis in the proximal LAD during the two months. (d) OCT findings before PCI in the proximal stenosis (arrow 1 in Figure 1(a)). (e) OCT findings before PCI in the distal stenosis (arrow 3 in Figure 1(a)). (f) OCT findings before PCI in the distal stenosis (arrow 2 in Figure 1(a)). (g) IVUS findings before PCI. RAO: right anterior oblique view; AP: anteroposterior; PCI: percutaneous coronary intervention; OCT: optical coherence tomography; IVUS: intravascular tomography; LAD: left anterior descending coronary artery. (From JC Cases 5: e83-e86, 2012 with permission).

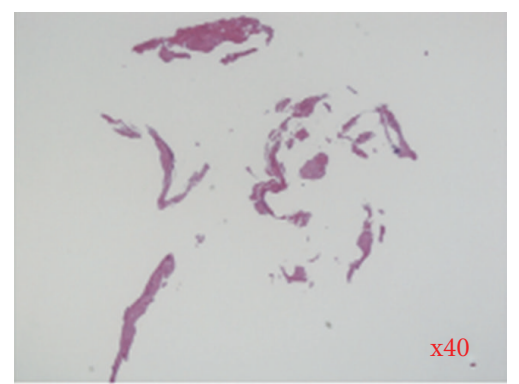

(a)

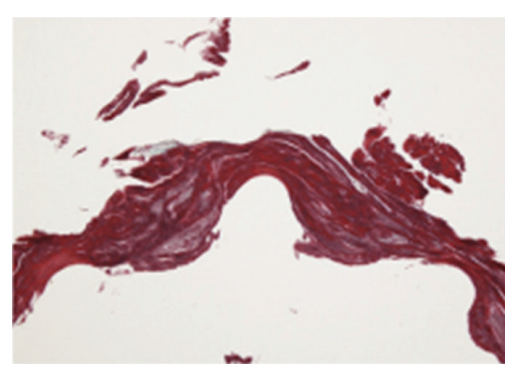

(d)

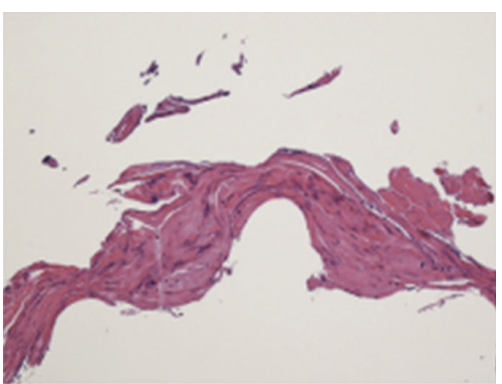

(b)

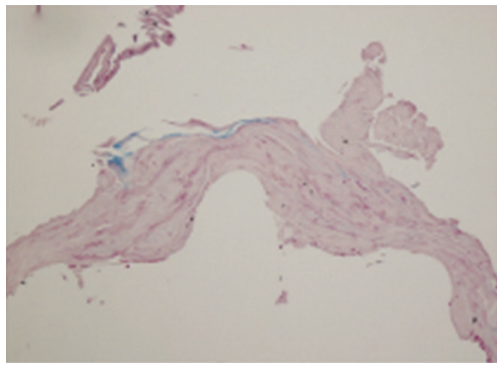

(e)

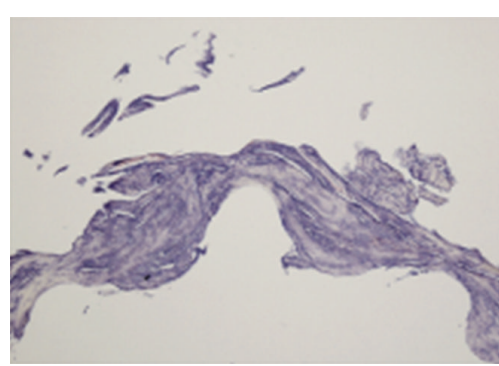

(c)

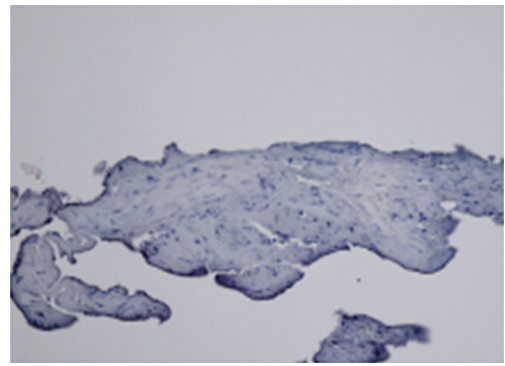

(f)

FIGURE 11: Materials retrieved on the Filtrap. After the procedure, the net of the Filtrap was cut and fixed in formalin. The main samples captured on the net were stained with hematoxylin-eosin staining, special staining, and immunohistological staining. (a) Microscopic findings of main retrieved samples. (b) Hematoxylin-eosin stain: $\times 200$. The retrieved samples consisted of hypocellular deposits and were mainly composed of fibrin deposits with a few inflammatory cells (neutrophils). (c) Phosphotungstic acid hematoxylin staining (PTAH): $\times 200$. Fibrin deposits were confirmed by PTAH staining as blue. (d) Masson trichrome staining: $\times 200$. Fibrin deposits were also confirmed by this special staining as red. (e) Alcian blue staining: $\times 200$. Proteoglycans were only slightly detected. (f) Immunoreactivity to SMC $\alpha$ actin: $\times 200$. Smooth muscle cells were not detected in this material. (From JC Cases 5:e83-e86, 2012 with permission). 
known. Takano et al. [8] demonstrated that expanded intraintima neovascularization may play a key role in atheroscrelotic progression and surrounding tissue instability, as well as intraplaque neovascularization of nonstent segments. In our pathological findings shown in the first case, there was sparse of neovascularization because material was retrieved only from superficial layer [4]. However, OCT detected microvessels near the stent struts. The trigger to develop microvessels into plaque should be elucidated, but there is a possibility that persistent chronic inflammation might be related to it. Inoue et al. [7] reported that autopsied samples after implantation of Palmaz-Schatz coronary stents (19 patients autopsied after noncardiac death 2-7 years poststenting) demonstrated that stainless steel stents evoked a remarkable foreign-body inflammatory reaction to the metal. In this study, they concluded that persistent peristrut chronic inflammatory cells might accelerate new indolent atherosclerotic changes and plaque vulnerability. We could not obtain material of peristent area; however, there was low signals surrounding some stent struts potentially indicating the presence of inflammation even 11 years after BMS deployment [4]. Autopsy studies have suggested that coronary thrombosis may occur in the absence of cardiac symptoms, resulting in progression of atherosclerosis [7]. Burke et al. [21] supported the hypothesis of plaque rupture as a mechanism of increased luminal narrowing. In that study, there was a low but significantly increased rate of cell proliferation in the SMC-rich regions of healed rupture sites, providing evidence of further plaque expansion. The matrix within the healed fibrous cap consists of a proteoglycanrich mass or a collagen-rich scar, depending on the healing phase. The pathological findings in this case seems to have similarity to that reported by Burke et al. [21]. Regarding the pathological mechanism of in-stent restenosis after 11 years, we speculate that persistent peristent chronic inflammation [6] might have yielded neovascularization accelerating new indolent atherosclerotic change [5] and consequent plaque vulnerability, which may have caused plaque ruptures or plaque erosions. After that, as healing process, proteoglycanrich mass associated with SMC narrowed the lumen as in the nonstented vessels.

\section{Relationships between Very Late Stent Thrombosis and In-Stent Restenosis}

Kang et al. [13] recently showed that, on OCT, in-stent neoatherosclerosis and intimal rupture were frequent in DES-treated patients with ISR. Miyazaki et al. [22] also showed that there was lipid-laden-like neointima involved in several cases of late ISR. Moreover, $36 \%$ of patients $(4 / 11)$ with lipid-laden-like neointima with disruption presented clinically with unstable angina. This result indicates that progression of lipid-laden-like neointima may promote the disruption and the subsequent late thrombosis, and that these processes may be overlapping in part in cases of late restenosis or late thrombosis. Also, a previous pathological study demonstrated that ISR was an additional risk factor for late stent thrombosis [23].

\section{Prevention and Management of Very Late In-Stent Restenosis}

It is well known that high cholesterol is related to the coronary events from Framingham study, and for the second prevention of cardiovascular events, lowering LDL cholesterol level up to $70 \mathrm{mg} / \mathrm{dL}$ is recommended according to some studies. However, it is still unknown whether neoatherosclerosis occurring in the BMS or DES is also related to the LDL level. To the best of our knowledge, there is no data showing the more progression of atherosclerosis in the nonstented area in patients with very late ISR. Although residual stent struts are reported to cause inflammation around the struts and work as trigger of neoatherosclerosis by histological studies, particular medical treatment for this phenomenon is not realistic right now. Instead, recent investigations focus on the development and improvement of bioresorbable vascular scaffolds [24-27] which resolve completely. Interventional strategy for ISR is still controversial. It must depend on the timing, pattern of ISR (BMS or DES) or focal, diffuse, proliferative, or occluded according to the Mehran classification [28]. In the cases with early ISR after BMS or DES, in which intimal hyperplasia is predominant factor, we usually use DES to prevent proliferation of smooth muscle cells. Paclitaxel-coated balloon angioplasty is not inferior to paclitaxel-eluting stents for BMS restenosis [29]. In ISR after DES implantation, a couple of studies have been conducted $[24,30,31]$. It should be determined whether same or the other DES is more beneficial in preventing recurrence of ISR. Recently, the PEPCAD-DES study revealed the superiority of paclitaxel-coated balloon angioplasty compared with plain balloon angioplasty for DES restenosis [32]. On the other hand, because mechanism of very late ISR is different from early ISR, the decision would be more complex. If weighing the inflammation caused by polymer is the cause of ISR, BMS or cutting balloon angioplasty would be recommended at the expense of superiority in suppressing smooth muscle cell proliferation with DES. DES implantation might induce additional inflammation during further followup period. If weighing the lesion morphology (diffuse, proliferative, and occluded) 1 or baseline risk factors (diabetes, hemodialysis, and so forth), DES could be the choice to prevent early ISR. Anyway, at the present time, no definite data are available, and case-based decision would be important to yield better clinical outcomes after additional PCI for very late ISR.

\section{Conflict of Interests}

The authors declare that they do not have a direct financial relation with the commercial identity mentioned in this paper that might lead to a conflict of interests for any of the authors.

\section{References}

[1] R. Choussat, C. Klersy, A. J. R. Black et al., "Long-term ( $>$ or $=$ years) outcome after Palmaz-Schatz stent implantation," American Journal of Cardiology, vol. 88, no. 1, pp. 10-16, 2001. 
[2] T. Kimura, K. Abe, S. Shizuta et al., "Long-term clinical and angiographic follow-up after coronary stent placement in native coronary arteries," Circulation, vol. 105, no. 25, pp. 2986-2991, 2002.

[3] K. Hasegawa, H. Tamai, E. Kyo et al., "Histopathological findings of new in-stent lesions developed beyond five years," Catheterization and Cardiovascular Interventions, vol. 68, no. 4, pp. 554-558, 2006.

[4] S. Ito, T. Yoshida, and S. Suzuki, "A case of extended late-phase in-stent restenosis after implantation of a bare-metal stent: intravascular ultrasound, optical coherence tomography and immunohistochemical findings," Journal of Invasive Cardiology, vol. 23, no. 4, pp. E86-E91, 2011.

[5] N. Gonzalo, P. W. Serruys, T. Okamura et al., "Optical coherence tomography patterns of stent restenosis," The American Heart Journal, vol. 158, no. 2, pp. 284-293, 2009.

[6] G. Nakazawa, F. Otsuka, M. Nakano et al., "The pathology of neoatherosclerosis in human coronary implants: bare-metal and drug-eluting stents," Journal of the American College of Cardiology, vol. 57, no. 11, pp. 1314-1322, 2011.

[7] K. Inoue, K. Abe, K. Ando et al., "Pathological analyses of long-term intracoronary Palmaz-Schatz stenting: is its efficacy permanent?" Cardiovascular Pathology, vol. 13, no. 2, pp. 109115,2004

[8] M. Takano, M. Yamamoto, S. Inami et al., "Appearance of lipid-laden intima and neovascularization after implantation of bare-metal stents. Extended late-phase observation by intracoronary optical coherence tomography," Journal of the American College of Cardiology, vol. 55, no. 1, pp. 26-32, 2009.

[9] S. Ito, K. Nakasuka, K. Miyata et al., "Ruptured plaque in a bare-metal stent 8 years after implantation-comparison of IVUS and OCT findings," Journal of Cardiology Cases, vol. 5, no. 1, pp. e1-e3, 2012.

[10] M. Habara, M. Terashima, K. Nasu et al., "Difference of tissue characteristics between early and very late restenosis lesions after bare-metal stent implantation: an optical coherence tomography study," Circulation, vol. 4, pp. 232-238, 2011.

[11] J. Cosgrave, S. J. Corbett, G. Melzi et al., "Late restenosis following sirolimus-eluting stent implantation," American Journal of Cardiology, vol. 100, no. 1, pp. 41-44, 2007.

[12] R. Virmani, G. Guagliumi, A. Farb et al., "Localized hypersensitivity and late coronary thrombosis secondary to a sirolimuseluting stent: should we be cautious?" Circulation, vol. 109, no. 6, pp. 701-705, 2004.

[13] S. J. Kang, G. S. Mintz, T. Akasaka et al., "Optical coherence tomographic analysis of in-stent neoatherosclerosis after drugeluting stent implantation," Circulation, vol. 123, no. 25, pp. 2954-2963, 2011.

[14] S. Ito, K. Nakasuka, K. Miyata et al., "Late in-stent restenosis after sirolimus-eluting stent implantation is related to thrmobus foramtion-insight from a case with IVUS, OCT, and histological findings," Journal of Cardiology Cases, vol. 5, no. 2, pp. e83-e86, 2012.

[15] S. Ito, K. Nakasuka, K. Morimoto et al., "Angiographic and clinical characteristics of patients with acetylcholine-induced coronary vasospasm on follow-up coronary angiography following drug-eluting stent implantation," Journal of Invasive Cardiology, vol. 23, no. 2, pp. 57-64, 2011.

[16] J. W. Kim, H. S. Seo, J. H. Park et al., "A prospective, randomized, 6-month comparison of the coronary vasomotor response associated with a zotarolimus- versus a sirolimuseluting stent. Differential recovery of coronary endothelial dysfunction," Journal of the American College of Cardiology, vol. 53, no. 18, pp. 1653-1659, 2009.
[17] T. Sawada, T. Shinke, J. Shite et al., "Impact of cytochrome $\mathrm{P} 4502 \mathrm{C} 19 * 2$ polymorphism on intra-stent thrombus after drug-eluting stent implantation in Japanese patients receiving clopidogrel," Circulation Journal, vol. 75, no. 1, pp. 99-105, 2011.

[18] K. Fujii, M. Masutani, and M. Ohyanagi, "Contribution of organized thrombus to in-stent restenosis after sirolimuseluting stent implantation: optical coherence tomography findings," European Heart Journal, vol. 29, no. 11, p. 1385, 2008.

[19] M. Takano, Y. Xie, D. Murakami et al., "Various optical coherence tomographic findings in restenotic lesions after sirolimus-eluting stent implantation," International Journal of Cardiology, vol. 134, no. 2, pp. 263-265, 2009.

[20] M. Nakano, F. Otsuka, and R. Virmani, "Optical coherence tomographic analysis of in-stent neoatherosclerosis after drugeluting stent implantation," Circulation, vol. 124, p. e955, 2011.

[21] P. Burke, F. D. Kolodgie, A. Falb et al., "Healed plaque ruptures and sudden coronary death Evidence that subclinical rupture has a role in plaque progression," Circulation, vol. 103, no. 7, pp. 934-940, 2001.

[22] S. Miyazaki, Y. Hyasa, T. Takahashi et al., "In vivo optical coherence tomography of very late drug-eluting stent thrombosis compared with late in-stent restenosis," Circulation Journal, vol. 76, no. 2, pp. 390-398, 2012.

[23] M. Joner, A. V. Finn, A. Farb et al., "Pathology of drug-eluting stents in humans. Delayed healing and late thrombotic risk," Journal of the American College of Cardiology, vol. 48, no. 1, pp. 193-202, 2006.

[24] H. G. Song, D. W. Park, Y. H. Kim et al., "Randomized trial of optimal treatment strategies for in-stent restenosis after drugeluting stent implantation," Journal of the American College of Cardiology, vol. 59, no. 12, pp. 1093-1100, 2012.

[25] G. G. Stefanini, R. A. Byrne, P. W. Serruys et al., "Biodegradable polymer drug-eluting stents reduce the risk of stent thrombosis at 4 years in patients undergoing percutaneous coronary intervention: a pooled analysis of individual patient data from the isar-test 3, isar-test 4, and leaders randomized trials," European Heart Journal, vol. 33, no. 10, pp. 1214-1222, 2012.

[26] Y. Onuma, T. Muramatsu, A. Kharlamov, and P. W. Serruys, "Freeing the vessel from metallic cage: what can we achieve with bioresorbable vascular scaffolds?" Cardiovascular Intervention and Therapeutics, vol. 27, no. 3, pp. 141-154, 2012.

[27] S. Nishio, K. Kosuga, K. Igaki et al., "Long-term (> 10 years) clinical outcomes of first-in-human biodegradable poly-1lactic acid coronary stents: Igaki-tamai stents," Circulation, vol. 125, pp. 2343-2353, 2012.

[28] R. Mehran, G. Dangas, A. S. Abizaid et al., "Angiographic patterns of in-stent restenosis: classification and implications for long-term outcome," Circulation, vol. 100, no. 18, pp. 1872-1878, 1999.

[29] M. Unverdorben, C. Vallbracht, B. Cremers et al., "Paclitaxelcoated balloon catheter versus paclitaxel-Coated stent for the treatment of coronary in-stent restenosis," Circulation, vol. 119, no. 23, pp. 2986-2994, 2009.

[30] G. Maluenda, I. Ben-Dor, M. A. Gaglia Jr et al., "Clinical outcomes and treatment after drug-eluting stent failure: the absence of traditional risk factors for in-stent restenosis," Circulation, vol. 5, no. 1, pp. 12-19, 2012.

[31] Y. G. Ko, J. S. Kim, B. K. Kim et al., "Efficacy of drug-eluting stents for treating in-stent restenosis of drug-eluting stents (from the korean des isr multicenter registry study [kiss])," 
American Journal of Cardiology, vol. 109, no. 5, pp. 607-613, 2012.

[32] H. Rittger, J. Brachmann, A. M. Sinha et al., "A randomized, multicenter, single-blinded trial comparing paclitaxel-coated balloon angioplasty with plain balloon angioplasty in drugeluting stent restenosis: the pepcad-des study," Journal of the American College of Cardiology, vol. 59, no. 15, pp. 1377-1382, 2012. 


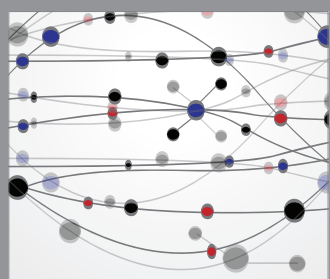

The Scientific World Journal
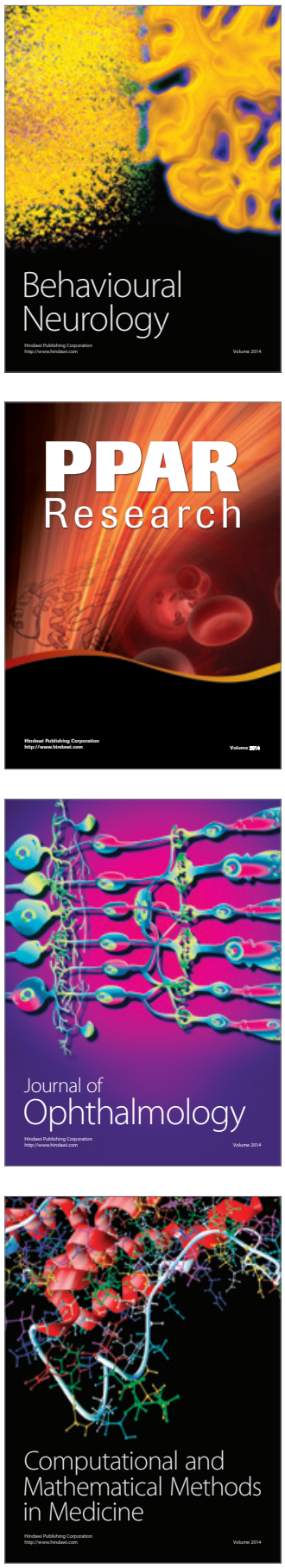

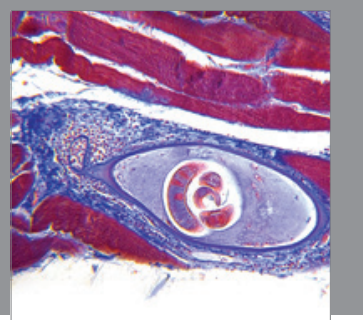

Gastroenterology

Research and Practice
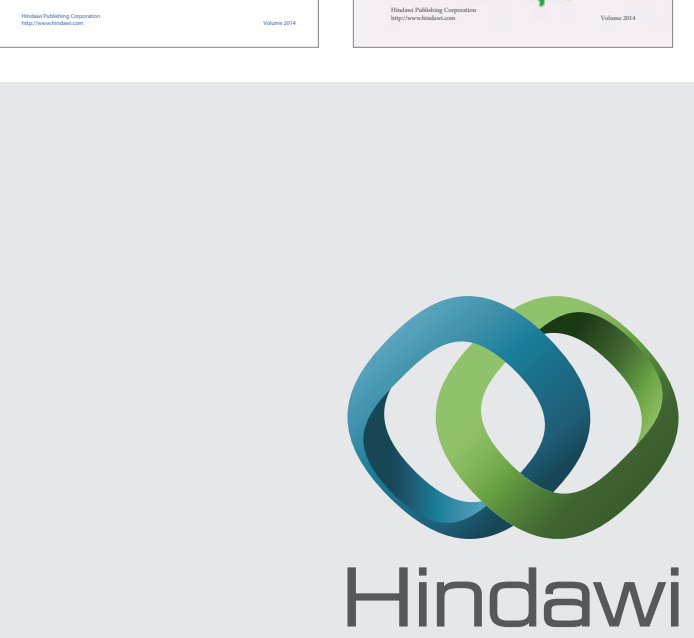

Submit your manuscripts at

http://www.hindawi.com
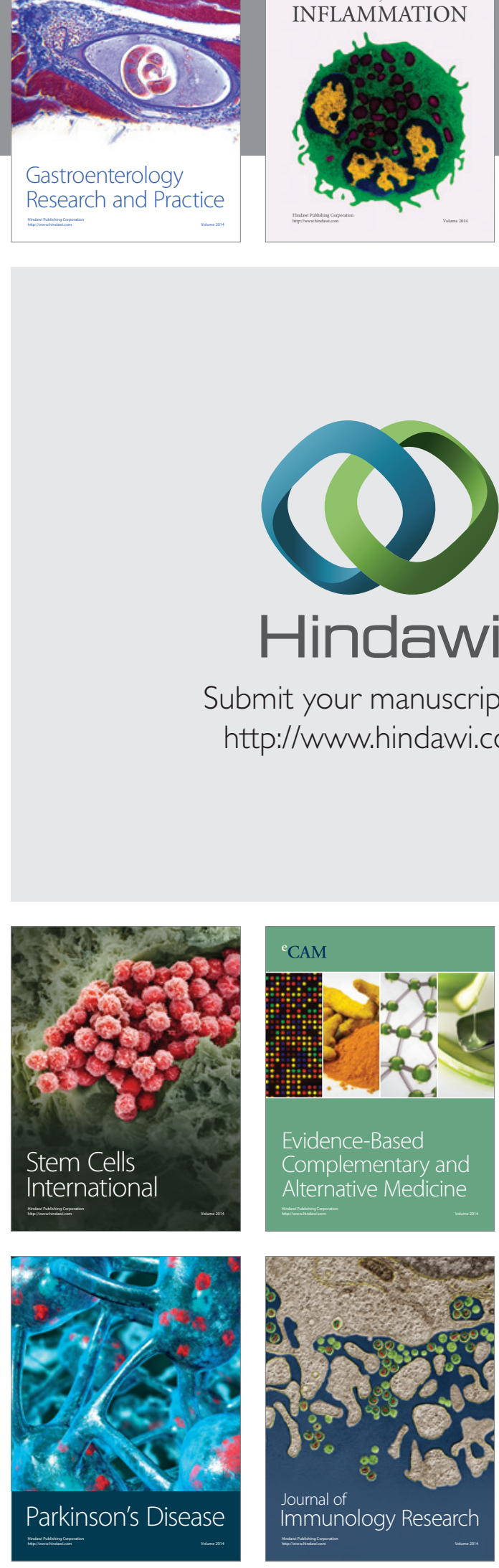

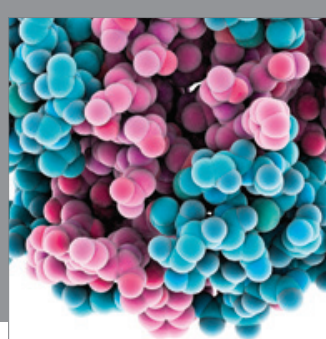

Diabetes Research
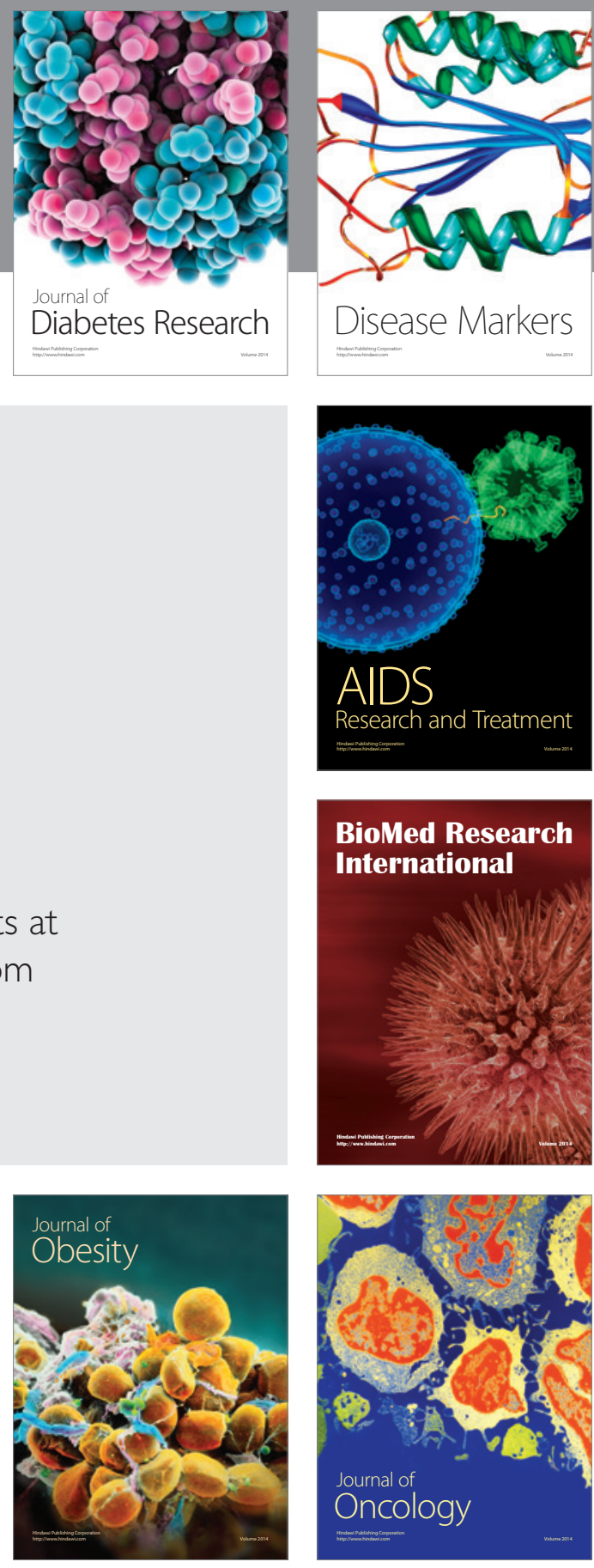

Disease Markers

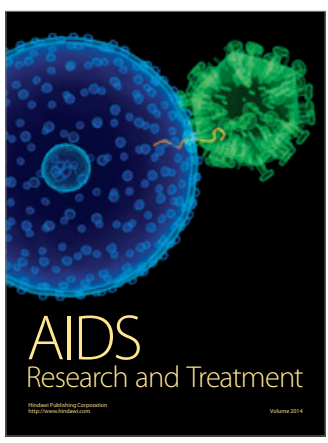

BioMed Research

International
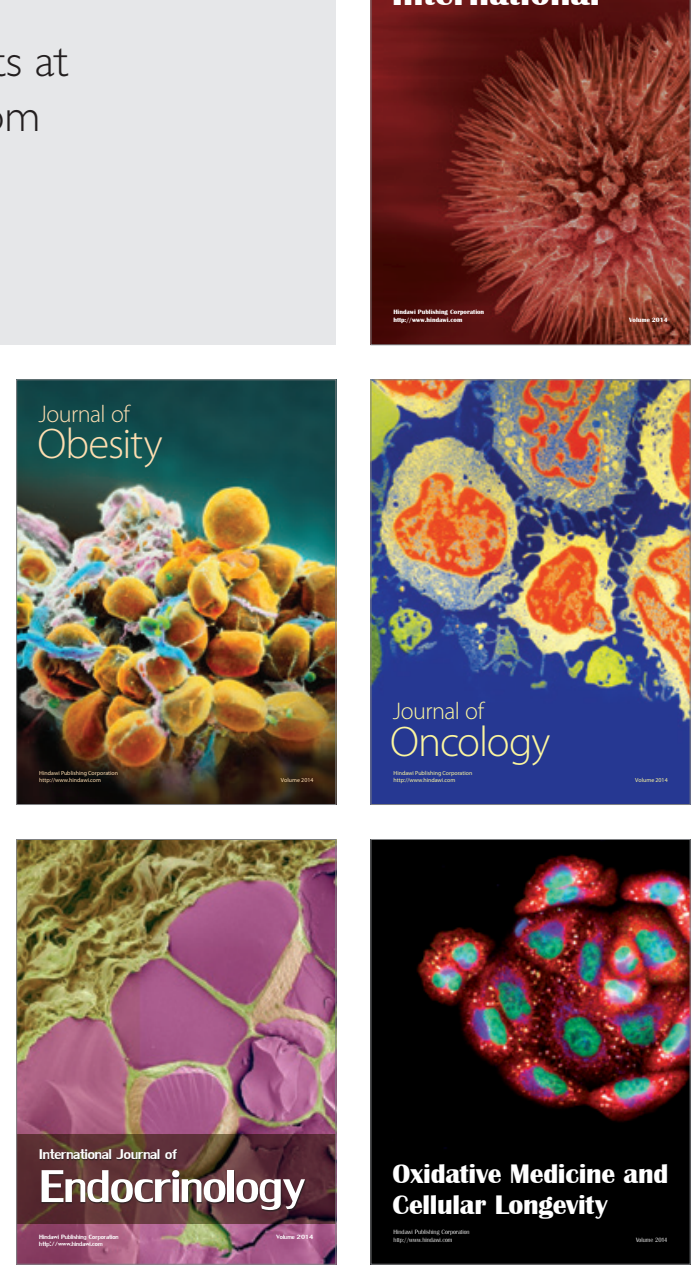